\title{
Influence des propriétés physico-chimiques du tétrafluorure d'uranium sur les modalités de la surveillance de l'exposition chronique à ce composé
}

\author{
E. ANSOBORLO*, B. GIBERT**, J. CHALABREYSSE*
}

(Manuscrit reçu le 22 septembre 1988)

RÉSUMÉ L'expérience acquise à Comurhex Narbonne dans la surveillance de l'exposition des travailleurs à des composés industriels de l'uranium nous a permis de développer une méthode d'évaluation des risques au poste de travail. Cette méthode appliquée à IUF $_{4}$ comporte 4 étapes :

1. la connaissance des caractéristiques physico-chimiques du composé (densité, surface spécifique, spectrométrie $X$ et enrichissement) ;

2. l'évaluation des concentrations au poste de travail ainsi que la granulométrie;

3. La solubilité in vitro dans des solutions biologiques synthétiques de type Gamble avec l'étude des effets de certains éléments comme l'oxygène ou les ions superoxydes $\mathrm{O}_{2}$; ceci permettra de définir la solubilité du composé en terme de dissolution suivant la classification $\operatorname{CIPR}(\mathbf{D}, \mathbf{W}, \mathbf{Y})$;

4. la survellance radiotoxicologique à l'aide d'examens de routine dans les urines et de compléments de mesures par le dosage des selles et par des anthropogammamétries.

Les résultats et données actuelles sont présentés.

ABSTRACT A method was developed in order to assess uranium exposure hazards at work stations based on the industrial experience acquired by Comurhex Malvesi at Narbonne. Applied to uranium tetrafluoride $\left(U_{4}\right)$, the method involves 4 steps :

1. characterization of the industrial compound, including its physico-chemical properties (density, surface area, $X$-ray spectrum and uranium enrichment);

2. assessment of work station concentrations and particle size distribution (AMAD):

3. in vitro biological solubility with different synthetic fluids such as Gamble solutions with different gases or compounds added (oxygen or superoxide ions $\mathrm{O}_{2}^{-}$) in order to determine the solubility class $D, W$ or $Y$;

4. workers' monitoring by routine measurements of urinary excretion completed, if necessary, by fecal excretion and $y$-spectrometry.

Results and present data on $U_{4}$ are presented.

* Commissariat à l'énergie atomique, Service d'hygiène industrielle, BP 38, 26701 Pierrelatte Cedex.

** Usine Comurhex Narbonne Malvesi, BP 222, Narbonne. 


\section{INTRODUCTION}

Le but de cette étude est de développer une méthodologie permettant l'évaluation des risques au poste de travail en corrélant l'exposition avec la surveillance radiotoxicologique du personnel. Cette méthodologie appliquée au composé $\mathrm{UF}_{4}$ dans un atelier de fluoration comporte principalement quatre étapes :

1. la connaissance du composé industriel, c'est-à-dire ses caractéristiques physico-chimiques;

2. l'étude de chaque poste de travail sur une période de 5 à 8 jours avec des mesures journalières de plusieurs types :

- prélèvements individuels pour estimer la fraction de poussières inhalables respirée,

- prélèvements en ambiance sur filtre pour valider les résultats obtenus avec les mesures journalières d'activité,

- mesures de granulométrie et détermination du diamètre aérodynamique médian en activité (DAMA) ;

3. l'étude de solubilité in vitro dans un liquide biologique synthétique afin de connaître la solubilité en terme de dissolution suivant la classification de la CIPR (D, W, Y) [4] ;

4. la surveillance du personnel de cette usine repose essentiellement sur les dosages d'uranium urinaire ; en examen de routine ces dosages sont pratiqués tous les 2 postes, avant la reprise du travail. Ces examens sont complétés, dans certains cas, par le dosage d'uranium dans les selles et par des anthropogammamétries. L'interprétation des résultats - plus ou moins délicate - s'appuie sur l'établissement de courbes d'élimination d'uranium urinaire et sur les courbes de concentration d'uranium dans l'air des ateliers. Cette interprétation doit aussi tenir compte de deux autres facteurs essentiels : la granulométrie des poussières uranifères et leur transférabilité.

\section{METHODES ET MATERIEL}

La méthodologie mise en oeuvre dans cette étude met en jeu plusieurs techniques qui sont décrites ci-dessous :

\section{- Caractéristiques physico-chimiques :}

Les mesures de densité sont effectuées au picnomètre dans un solvant et la surface spécifique est déterminée par la méthode du BET. La mesure aux rayons $X$ est faite sur un appareil Phillips et la détermination des angles de diffraction permet d'obtenir une identification des composés. Enfin, la composition isotopique est obtenue par spectrométrie de masse sur l'échantillon même de poussière d'UF ${ }_{4}$. 


\section{- Prélèvements d'atmosphère :}

Les prélèvements individuels sont effectués à l'aide d'une pompe Dupont P4000 suivant la norme Afnor NF X 43-256 sur cassettes millipores de diamètre $25 \mathrm{~mm}$ et sur filtres millipores $0,8 \mu \mathrm{m}$. La fraction ainsi collectée correspond à la fraction inspirable. Le débit d'inspiration est de 1 I. $\mathrm{min}^{-1}$.

Les prélèvements en ambiance sont faits sur filtre millipores $0,8 \mu \mathrm{m}$ avec un débit de $30 \mathrm{l} \cdot \mathrm{min}^{-1}$ assuré par des pompes millipores. Ces prélèvements sont ainsi comparés avec les mesures journalières effectuées pour le contrôle d'activité sur chaque poste. Les analyses des filtres sont réalisées par comptage $\alpha$ et exprimées en becquerels par mètre cube.

\section{- Granulométrie :}

Ces mesures sont effectuées à l'aide d'un impacteur à cascade Andersen à 8 étages (Mark II) avec un débit de $281 . \mathrm{min}^{-1}$ et les résultats sont exprimés sous forme de distribution massique avec détermination du diamètre aérodynamique médian en activité (DAMA) ainsi que la déviation géométrique standard $\sigma \mathrm{g}$.

\section{- Etude de solubilité in vitro dans un liquide biologique synthétique :}

Le test de solubilité retenu pour cette étude est le test statique décrit par KANAPILLY [8, 9], KALKWARF [7] et ANSOBORLO [3]. Le principe de base est un contact direct de la poussière avec un liquide biologique synthétique ou solvant. Dans cette étude les solvants retenus ont été : (1) les carbonates $\left(\mathrm{NaHCO}_{3} 27 \mathrm{mmole}^{-1}\right)$, (2) la solution de Gamble [5], (3) Gamble avec barbotage d'oxygène, et enfin (4) Gamble additionnée de pyrogallol (P) $\left(0,8 \mathrm{~g} \mathrm{I}^{-1}\right)$, de superoxyde dismutase (SOD) $\left(0,075 \mathrm{~g} \mathrm{I}^{-1}\right)$ avec barbotage d'oxygène.

L'idée d'addition de pyrogallol, d'oxygène et de SOD à la solution de Gamble est venue de l'étude des mécanismes de dissolution au niveau du macrophage alvéolaire décrits par MARKLUND [10] et JADOT [6] : en effet, ces auteurs montrent l'apparition au niveau du macrophage alvéolaire d'ion $\mathrm{O}_{2}^{-}$jouant un rôle d'oxydant puissant et de SOD modérant cette action. Ce phénomène a été reproduit in vitro en utilisant le pyrogallol qui, sous l'action de l'oxygène, donne naissance aux ions $\mathrm{O}_{2}^{-}$.

Lors de ces essais, les échantillons sont prélevés journalièrement et analysés par fluorimétrie à l'aide d'un appareil à fluorescence $X$ Schlumberger (modèle CEA). Les résultats sont exprimés en pourcentage d'uranium cumulé non dissous en fonction du temps.

\section{- Méthode d'évaluation de la contamination interne :}

a) Excreta (urines, selles)

- Teneurs pondérales par fluorimétrie

- directement : limite de détection $4 \mu \mathrm{g} \mathrm{I}^{-1}\left(3 \mu \mathrm{g} \mathrm{g}^{-1}\right.$ de créatinine),

- après extraction chimique : limite de détection $0,1 \mu \mathrm{g} \mathrm{I}^{-1}$ (urines $0,07 \mu \mathrm{g} \mathrm{g}^{-1}$ de créatinine, selles $0,1 \mu \mathrm{g}$ ) ; 
- Activité spécifique :

Une séparation chimique de l'uranium est opérée à l'aide d'une résine liquide AMBERLITE LA2 : sur l'éluat on procède à une mesure de l'activité. Les sensibilités sont les suivantes : urines : $10 \mathrm{mBq} \mathrm{^{-1 }}$ soit $7 \mathrm{mBq} \mathrm{g}{ }^{-1}$ créatinine, selles : $4 \mathrm{mBq} / \mathrm{selles} 24 \mathrm{~h}$.

\section{b) Anthropogammamétrie}

A Pierrelatte, nous disposons d'une cellule de plomb dont la détection est assurée par deux détecteurs de type PHOSWICH. Les mesures sont effectuées sous les aisselles. Pour un examen d'une durée de $20 \mathrm{~min}$, la limite de détection est de $150 \mathrm{~Bq}(4 \mathrm{nCl})$. Cette technique n'est pas utilisée dans le cas de IUFF $_{4}$ en raison de son caractère transférable mais, par contre, s'avère nécessaire en ce qui concerne l'étude de composés moins solubles.

L'atelier de fluoration étudié comporte principalement deux postes de travail : un poste appelé "prise d'essai" avec prélèvement des échantillons pour le suivi en analyse de I'UF ${ }_{4}$ et un poste "échantillonnage" pour la mise en fûts de cet $\mathrm{UF}_{4}$. Un opérateur équipé d'un appareil de prélèvement individuel surveille ces deux postes.

\section{RÉSULTATS}

Les résultats obtenus sont présentés dans l'ordre de la méthodologie décrite précédemment et comportent quatre observations principales résumées sous forme de tableaux et figures.

Les tableaux I et II présentent des résultats correspondant d'une part aux caractéristiques physico-chimiques et granulométriques des poussières d'UF ${ }_{4}$ (tableau I) et d'autre part aux concentrations journalières mesurées sur 5 jours aux postes de travail étudiés (tableau II).

TABLEAU 1

Caractéristiques physico-chimiques de l'UF 4 de l'usine Comurhex de Malvési

\begin{tabular}{|c|c|c|c|c|c|c|}
\hline $\begin{array}{l}\text { Lieu } \\
\text { ou } \\
\text { poste }\end{array}$ & $\begin{array}{c}\text { Granulométrie } \\
\text { Andersen } \\
\text { Dama }(\mu \mathrm{m})\end{array}$ & $\begin{array}{l}\text { Granul } \\
\text { Comptag } \\
\text { D } \\
\text { géométrique }\end{array}$ & $\begin{array}{l}\text { métrie } \\
\text { Danuel } \\
\text { Dama }\end{array}$ & $\begin{array}{l}\text { Densité } \\
\left(\mathrm{g} \mathrm{cm}^{-3}\right)\end{array}$ & $\begin{array}{l}\text { Surface } \\
\text { spécifique } \\
\left(\mathrm{m}^{2} \mathrm{~g}^{-1}\right)\end{array}$ & Spectre \\
\hline $\begin{array}{c}\text { Prise } \\
\text { d'essai }\end{array}$ & $\begin{array}{c}\text { dae }=6,8 \mu \mathrm{m} \\
o \mathrm{~g}=2,5 \\
33 \% \text { dae } \\
<5 \mu \mathrm{m}\end{array}$ & $\begin{array}{c}\mathrm{dg}=2,8 \mu \mathrm{m} \\
\sigma=2,1 \\
80 \% \mathrm{dg} \\
<5 \mu \mathrm{m}\end{array}$ & $\begin{array}{c}\mathrm{dae}=6,9 \mu \mathrm{m} \\
\sigma \mathrm{g}=2,1 \\
33 \% \text { dae } \\
<5 \mu \mathrm{m}\end{array}$ & $\mathrm{d}$ app $=2.8$ & \multirow[t]{3}{*}{1,6} & \multirow{3}{*}{$\mathrm{UF}_{4}$} \\
\hline $\begin{array}{c}\text { Echantillon- } \\
\text { nage }\end{array}$ & $\begin{array}{c}\text { dae }=9 \mu \mathrm{m} \\
\sigma g=2,4 \\
24 \% \text { dae } \\
<5 \mu n\end{array}$ & $\begin{array}{c}\mathrm{dg}=2,6 \mu \mathrm{m} \\
\sigma \mathrm{g}=2,3 \\
78 \% \mathrm{dg} \\
<5 \mu \mathrm{m}\end{array}$ & $\begin{array}{c}\text { dae }=6,4 \mu \mathrm{m} \\
\sigma \mathrm{g}=2,3 \\
30 \% \mathrm{dae} \\
<5 \mu \mathrm{m}\end{array}$ & d réel $=6$ & & \\
\hline Individuel & & $\begin{array}{c}\mathrm{dg}=3,3 \mu \mathrm{m} \\
\sigma g=2,9 \\
64 \% \mathrm{dg} \\
<5 \mu \mathrm{m}\end{array}$ & $\begin{array}{c}\text { dae }=8,1 \mu \mathrm{m} \\
\sigma \mathrm{g}=2,9\end{array}$ & & & \\
\hline
\end{tabular}


TABLEAU \|

Mesure de concentration en uranium aux postes de travail de l'atelier de fluoration $\left(\mathrm{Bq} \mathrm{m}^{-3}\right)$

\begin{tabular}{|c|c|c|c|c|}
\hline Date & Prise d'essai & Echantillonnage & APA $\left(^{*}\right)$ & Individuel \\
\hline $06 / 06 / 88$ & 0,93 & 0,37 & 0,52 & 1,63 \\
$07 / 06 / 88$ & 0,84 & 0,85 & 0,20 & 0,1 \\
$08 / 06 / 88$ & 3,69 & 1,08 & 0,19 & 0,77 \\
$09 / 06 / 88$ & 0,68 & 1,13 & 0,15 & 0,62 \\
$10 / 06 / 88$ & 0,1 & 2,86 & 0,25 & 0,5 \\
\hline
\end{tabular}

* APA : Appareil de prélèvement d'air.

Dans le tableau I, le résultat principal est la mesure du DAMA sur les deux postes "prise d'essai" et "échantillonnage" ainsi qu'en prélèvement individuel. Dans ces trois cas, ce DAMA est compris entre 6,4 et $9 \mu \mathrm{m}$ avec des déviations standards géométriques $(\sigma g)$ supérieures à 2 (distribution non homogène). Notons aussi qu'en moyenne $30 \%$ des particules ont un DAMA inférieur à $5 \mu \mathrm{m}$.

Dans le tableau II, nous trouvons les concentrations d'uranium sous forme d'UF $F_{4}$, mesurées aussi bien par prélèvements en ambiance et APA (appareil de prélèvement d'air) appartenant aux services de sécurité de l'usine de Malvési que par prélèvements individuels du 6 au 10 juin 1988. Ces valeurs sont comprises entre 0,10 et $3,69 \mathrm{~Bq} \mathrm{~m}^{-3}$ en ambiance et vont de 0,15 à $0,52 \mathrm{~Bq} \mathrm{~m}^{-3}$ pour les APA et de 0,10 à $1,63 \mathrm{~Bq} \mathrm{~m}^{-3}$ en individuel.

Le tableau III et la figure 1 présentent les résultats obtenus lors des essais in vitro comportant plusieurs solutions. Ces résultats sont exprimés sous la forme : avec

$\%$ uranium cumulé non dissous $=\sum_{i=1}^{n} A i \exp (-0.693 \mathrm{t} / \mathrm{Ti})$

$A i=\%$ initial $d u$ composé $i$ et $\Sigma A i=1$

$\mathrm{Ti}=$ temps de demi-dissolution du composé $\mathrm{i}$.

TABLEAU III

Résultats des essais de solubilité in vitro de l'UF 4 suivant les classes $D, W, Y$ de la CIPR

\begin{tabular}{|c|c|c|c|c|c|c|c|c|}
\hline Solvant & \multicolumn{2}{|c|}{$\stackrel{1}{\mathrm{NaHCO}_{3}}$} & \multicolumn{2}{|c|}{$\mathrm{G}^{2}\left(^{*}\right)$} & \multicolumn{2}{|c|}{$\mathrm{G}+{ }^{3} \mathrm{O}_{2}$} & \multicolumn{2}{|c|}{$\begin{array}{c}{ }^{4} \stackrel{4}{+} \mathrm{G}+ \\
\text { SOD }\left(^{(*)}+\mathrm{O}_{2}\right.\end{array}$} \\
\hline CIPR & Al $(\%)$ & Ti (d) & Al (\%) & $\mathrm{Ti}(\mathrm{d})$ & Ai (\%) & $\mathrm{Ti}$ (d) & Ai $(\%)$ & $\mathrm{Ti}(\mathrm{d})$ \\
\hline $\begin{array}{l}D \\
W \\
Y\end{array}$ & 100 & 11 & 100 & 285 & 100 & 26 & 100 & 1.5 \\
\hline
\end{tabular}

* $\mathrm{G}=$ Gamble $; \mathrm{P}=$ pyrogallol $; \mathrm{SOD}=$ Superoxyde dismutase. 


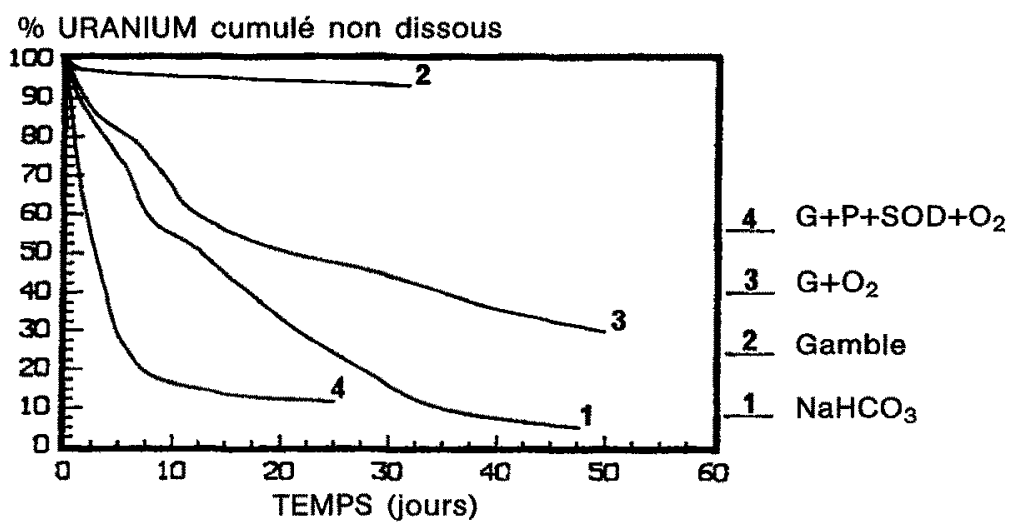

Fig. 1. - Résultats des essais de solubllité in vitro sur UF4.

Cette définition permet de rapporter chaque cinétique à la classification $D, W, Y$ de la CIPR [4].

Nous constatons, dans ce tableau III, que la solution de Gamble seule (2) donne l'UF ${ }_{4}$ comme un composé insoluble de classe $Y$, résultat déjà obtenu par ANSOBORLO [2]. En présence de carbonates [1], et de Gamble $+\mathrm{O}_{2}, \mathrm{UF}_{4}$ prend un comportement $\mathrm{D}-\mathrm{W}$ à W [3]. Enfin, sous l'action des ions superoxydes $\mathrm{O}_{2}^{-}$et de la SOD (4), $\mathrm{L}^{\prime} \mathrm{UF}_{4}$ a un comportement de type $D$ avec une période de demi-dissolution de 1,5 jour.

Le résultat se rapproche des essais in vitro réalisés par ANDRÉ et MÉTIVIER [1] et STRADLING [12], s'appuyant sur des expérimentations animales (essais sur rats et singes par instillation et inhalation) ainsi que des essais in vitro sur cultures cellulaires dont la conclusion est l'appartenance d'UF $_{4}$ à la classe $D$.

Enfin, les figures 2 et 3 représentent l'évolution des concentrations atmosphériques et urinaires de 1977 à 1988. Ces deux fonctions sont décroissantes dans le temps, sans corrélation significative entre elles, notamment au niveau des pics.

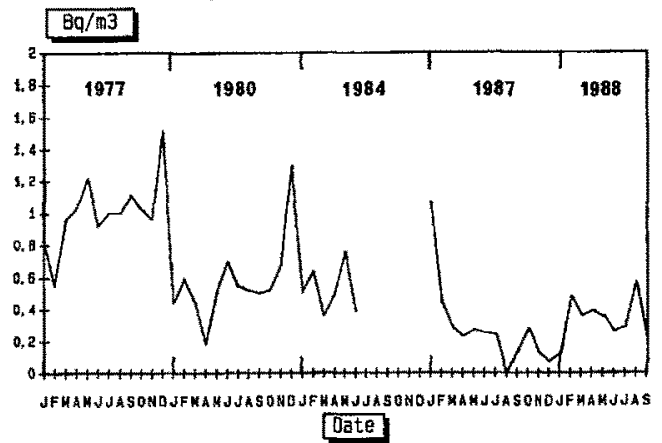

Fig. 2. - Evolution de la concentration atmosphérique en uranium de 1977 à 1988 dans l'atelier de fluoration. 


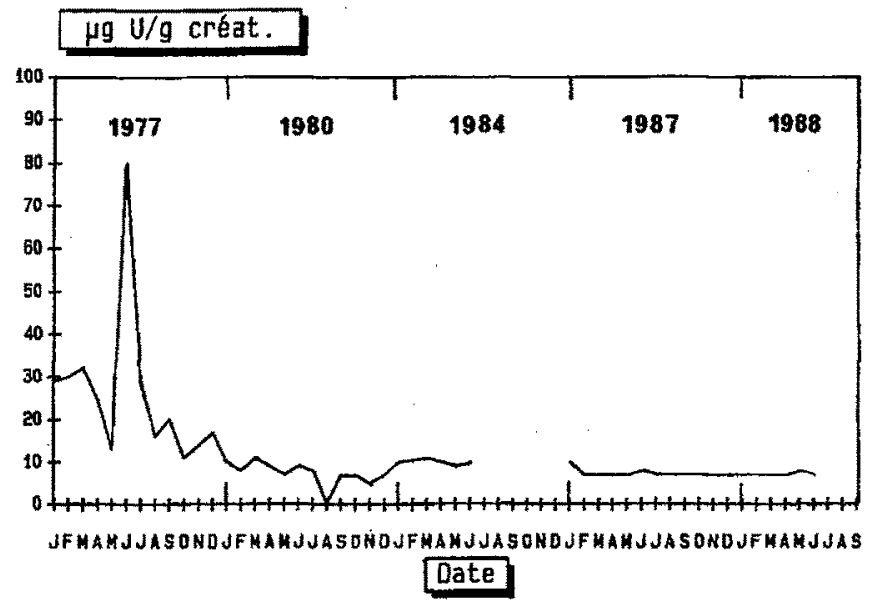

Fig. 3. - Evolution de la concentration urinaire en uranium de 1977 à 1988 dans P'atelier de fluoration.

L'observation fréquente de 1977 à 1980 de pics urinaires importants suivis d'une décroissance rapide corrobore les résultats in vitro et in vivo classant I'UF $_{4}$ comme un élément transférable de classe $D$.

\section{DISCUSSION}

Cette méthodologie a été développée pour l'UF 4 qui avait déjà fait l'objet d'études précédentes.

Dans l'atelier où les prélèvements ont été faits pour l'étude présente, I'UF $_{4}$ se présentait à l'état pur. Les concentrations mesurées aussi bien en ambiance qu'en individuel doivent être comparées à la LDCA (limite de concentration admissible dans l'air) définie dans le décret 88-662 du 8 mai 1988. Si à la suite des essais in vitro obtenus dans cette étude et en tenant compte des résultats similaires obtenus par ANDRÉ et MÉTIVIER [1] ainsi que par STRADLING [12], on considère que UF $_{4}$ est un composé de type $D$, la LDCA correspondante est de $20 \mathrm{~Bq} \mathrm{~m}^{-3}$.

Les valeurs de concentrations obtenues dans l'atelier fluoration sont très inférieures à $20 \mathrm{~Bq} \mathrm{~m} \mathrm{~m}^{-3}$ puisque la moyenne en ambiance sur 5 jours est de $1,2 \mathrm{~Bq} \mathrm{~m}^{-3}$ et de $0,7 \mathrm{~Bq} \mathrm{~m}^{-3}$ en individuel. Notons que ces prélèvements individuels, qui sont les plus représentatifs de l'exposition réelle, donnent des valeurs de concentrations sensiblement plus élevées que I'APA. D'autre part, les résultats de granulométrie montrent que, dans l'ensemble, seulement $30 \%$ des particules ont un DAMA inférieur à $5 \mu \mathrm{m}$ et que le DAMA moyen est compris entre 6,4 et $9 \mu \mathrm{m}$.

En ce qui concerne les tests in vitro, notons l'importance de l'influence des ions superoxydes qui ont permis d'oxyder l'uranium de la valence 4 à la valence 6 , expliquant ainsi la grande solubilité de ce composé comparativement à la solution de Gamble dans laquelle ce 
mécanisme est inhibé par la présence des phosphates. Cette oxydation a aussi lieu en présence d'oxygène seul, mais plus faiblement. Ce résultat est très important et traduit le rôle essentiel des tests in vitro dans la compréhension des mécanismes de dissolution.

Si nous considèrons le modèle de la CIPR repris par PIECHOWSKI [11], nous constatons que, pour un tel DAMA, correspond la répartition suivante dans l'arbre respiratoire : environ $80 \%$ dans le naso-pharynx (NP), $8 \%$ dans l'arbre trachéo-bronchique (TB) et $7 \%$ dans le parenchyme pulmonaire $(P)$ (les pourcentages sont exprimés en fraction de la quantité initiale inhalée). Tenant compte de cette répartition liée à la granulométrie des particules inhalées et considérant les résultats in vitro et in vivo donnant l'UF 4 comme un composé transférable de classe $D$, nous pouvons - à partir du schéma du métabolisme d'un radionucléide - prédire le comportement du composé étudié. Ainsi, pour un composé D dont la répartition aux portes d'entrée est de $88 \%$ entre NP et TB et dont les périodes d'épuration sont très rapides $(0,01$ j pour NP et TB et 0,5 j pour $P$ ), on peut considérer qu'une très grande partie sera éliminée via le liquide extra-cellulaire par les uffines avec une période très rapide. Cette modélisation suivant PIECHOWSKI correspond bien aux résultats observés entre 1977 et 1980 , lorsque les concentrations urinaires en uranium étaient assez fortes et correspondaient à de forts pics instantanés, suivis de décroissances très rapides.

L'utilisation d'un masque de protection du type Home ergonomique tel qu'il est présenté dans la figure 4 n'a pas été jugé nécessaire dans un tel atelier, attendu que les concentrations sont très en dessous de la LDCA et que le masque classique (type filtre) semble suffisant si l'on considère les résultats en concentration urinaire. Par contre, notons l'intérêt d'un tel masque dans le cas de composés non transférables de classe $Y$ pour lesquels la LDCA est beaucoup plus restrictive $(0,65 \mathrm{~Bq} \mathrm{~m}-3)$.

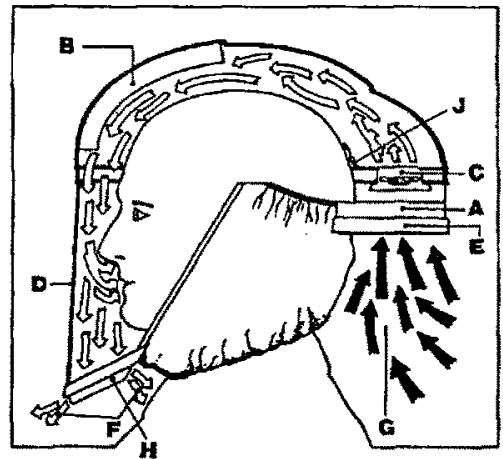

A - Filtre principal ; B - Batterie ; C - Ensemble moteur et ventilateur ;

$D$ - Ensemble visière et col ; E - Préfiltre ; F - Sortie d'air ; G - Entrée d'air vicié ; $H$ - Valve ; J - Interrupteur.

Fig. 4. - Appareil de protection respiratoire à ventilation intégrée. 


\section{CONCLUSION}

Les difficultés d'interprétation rencontrées lors de l'examen de certains graphiques, l'absence de corrélation entre certaines courbes d'élimination urinaire et de concentration d'uranium dans l'air des ateliers nous ont amenés à nous poser de nouvelles questions sur les caractéristiques physico-chimiques des composés et surtout sur la période biologique effective des poussières inhalées, ce qui doit conditionner les normes de surveillance des ouvriers.

Cette méthodologie, décrite précédemment dans le cas de l'UF 4 nous

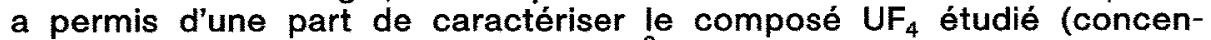
tration comprise entre 0,1 et $3,7 \mathrm{~Bq} \mathrm{~m}^{-3}$ et un DAMA entre 6,4 et $9 \mu \mathrm{m}$ ) et d'autre part à l'aide de tests in vitro développés avec différents solvants de déterminer, en comparaison avec des tests in vivo par inhalation, son comportement en terme de dissolution (composé transférable de type D). Ces résultats, parmi lesquels il faut souligner l'importance des essais in vitro qui ont permis de comprendre les mécanismes de dissolution, semblent très bien corrélés avec les observations de suivis urinaires pratiqués entre 1977 et 1988.

Ainsi les mesures de concentrations, couplées aux mesures urinaires, aux caractéristiques physico-chimiques et aux études de solubilité in vitro ont permis de bien cerner le problème et d'établir un mode de surveillance efficace qui s'est traduit par une très grande amélioration des conditions de travail au fil des années.

\section{BIBLIOGRAPHIE}

[1] ANDRÉ S., METIVIER H., AUGET D., LANTENOIS G., BOYER M., MASSE R. Assessment of uranium tetrafluoride dissolution in the lung by in vivo and in vitro methods. In : Biological assessment of occupational exposure to actinides, Versailles, May 30 - June 2, 1988. Radiat. Prot. Dos., 1989, 26 (1/4) 75-81.

[2] ANSOBORLO E. - Etude des caractéristiques physico-chimiques de composés uranifères sur des postes de travail - Thèse de docteur ingénieur ECAM, 1983.

[3] ANSOBORLO E., CHALABREYSSE J. - Etude de l'influence de divers paramètres sur la solubilité in vitro de composés industriels ou diuranates d'ammonium calcinés. Radioprotection, 1989, 24 (1) 3-12.

[4] COMMISSION INTERNATIONALE DE PROTECTION RADIOLOGIQUE (CIPR). Limits for intakes of radionuclides by workers (ICRP publication 30). Oxford : Pergamon Press, 1979.

[5] GAMBLE J.L. - Chemical anatomy, physiology and pathology of extracellular fluid, 8th edition. Harvard university press, 1967, pp. 4-11.

[6] JADOT G. - Les superoxydes dismutases, biochimie, pharmacologie, thérapeutique. Paris : Masson, 1988.

[7] KALKWARF D.R. - Solubility classification of airbone uranium products from LWR fuel plants. Rapport NUREG/CR-1428 (PNL-3411), 1980. 
[8] KANAPILLY G.M., GOH C.H.T. - Some factors affecting the in vitro rates of dissolution of respirable particles of relatively low dissolution. Health Phys., $1973,25,225-237$.

[9] KANAPILLY G.M., RAABE O.G. - Measurement of in vitro dissolution of aerosol particles for comparison to in vivo dissolution in the lower respiratory tract after inhalation. Health Phys., 1973, 24, 497-507.

[10] MARKLUND S., MARKLUND G. - Involvement of the superoxide anion radical in the auto-oxidation of pyrogallol and a convenient assay for superoxide dismutase. Europ. J. Biochem, 1974, 47, 469-474.

[11] PIECHOWSKI J., MENOUX B. - Rétention et excrétion des radionucléides après incorporation par inhalation chez l'homme adulte. Rapport CEAR-5266, 1984.

[12] STRADLING G.N., STATHER J.W., STRONG J.C., SUMNER S.A., TOWNDROW C.G., MOODY J.C., LENNOX A., SEDGWICK D., COOKE N. - Metabolism of some industrial uranium tetrafluorides after deposition in the rat lung. Human Toxicol, $1985,4,159-168$. 